\title{
Failure to improve after ovarian resection could be a marker of recurrent ovarian teratoma in anti-NMDAR encephalitis: a case report
}

This article was published in the following Dove Press journal:

Neuropsychiatric Disease and Treatment

\author{
Yuto Uchida' \\ Daisuke Kato' \\ Yoriko Yamashita ${ }^{2}$ \\ Yasuhiko Ozaki ${ }^{3}$ \\ Noriyuki Matsukawa' \\ 'Department of Neurology, \\ ${ }^{2}$ Department of Experimental \\ Pathology, ${ }^{3}$ Department of \\ Obstetrics and Gynecology, Nagoya \\ City University Graduate School of \\ Medical Sciences, Nagoya, Japan
}

Correspondence: Daisuke Kato

Department of Neurology, Nagoya City

University Graduate School of Medical

Sciences, I Kawasumi, Mizuho-ku,

Nagoya 467-860I, Japan

$\mathrm{Tel}+8 \mathrm{I} 528538094$

Fax +8I 528523590

Email daisuke@med.nagoya-cu.ac.jp

\begin{abstract}
Anti-N-methyl-D-aspartate receptor (NMDAR) encephalitis is a type of autoimmune encephalitis that can be paraneoplastic and usually responds to tumor resection and immunotherapy. More than $75 \%$ of patients with anti-NMDAR encephalitis fully recover or have only mild sequelae, whereas the remainder experience severe disability. It remains unknown why certain cases have refractory clinical disease courses. We report a case of anti-NMDAR encephalitis with bilateral ovarian teratomas who was refractory to tumor resection and early initiation of immunotherapy. During intensive care, immunohistochemical analyses of her cerebrospinal fluid showed persistently high reactivity of NMDAR antibody over time. Six months after the operation, pelvic computed tomography detected a recurrent ovarian teratoma. After total enucleation of the bilateral ovaries, with significant pathological findings of bilateral mature cystic teratomas, her clinical condition improved rapidly, paralleled by a decrease in anti-NMDAR reactivity. This case illustrates the need to keep considering why extensive treatment fails to influence the disease when we encounter patients with refractory anti-NMDAR encephalitis. Failure to improve after ovarian resection could be a marker of recurrent ovarian teratoma in anti-NMDAR encephalitis.
\end{abstract}

Keywords: N-methyl-D-aspartate receptor, antibody, autoimmune encephalitis, refractory, recurrence

\section{Introduction}

Anti-N-methyl-D-aspartate receptor (NMDAR) encephalitis, formally recognized in 2007 in women with ovarian or mediastinum teratomas, ${ }^{1}$ has been increasingly described as a significant cause of autoimmune and paraneoplastic encephalitis., ${ }^{2,3}$ Patients with anti-NMDAR encephalitis develop prominent psychiatric symptoms, followed by seizures, disturbance of consciousness, and autonomic instability, often requiring intensive care. ${ }^{2,3}$ The presence of antibodies against the NR1/NR2 heteromers of NMDAR in cerebrospinal fluid (CSF) and serum confirms the diagnosis, ${ }^{1}$ which should urge clinicians to search for an underlying neoplasm.

Approximately half the adult patients with anti-NMDAR encephalitis have tumors, most commonly ovarian teratomas, ${ }^{4}$ and should be promptly treated with tumor resection and immunotherapy. Over $75 \%$ of patients fully recover or have only mild sequelae, whereas the remainder experiences substantial deficits or death. ${ }^{2}$

However, it is unknown why certain cases have refractory clinical courses, even with prompt initiation of treatment, which is the most important prognostic factor. ${ }^{5}$ In such patients, persistently high levels of NMDAR antibody might correlate with their lack of improvement. ${ }^{6}$ We report the case of a patient with anti-NMDAR encephalitis 
with bilateral ovarian teratomas who was refractory to tumor resection and early initiation of immunotherapy, presenting with chronic anti-NMDAR reactivity. After the bilateral ovaries were totally enucleated in a second operation, with significant pathological findings of recurrent bilateral mature cystic teratomas, the patient has rapidly improved, paralleled by a decrease in anti-NMDAR reactivity. To the best of our knowledge, this is the first case report presenting the successful reoperative therapy for recurrent bilateral ovarian teratomas in a patient with anti-NMDAR encephalitis.

\section{Case report}

A 20-year-old woman had experienced headaches and low-grade fever for a week, followed by acute, progressive disturbance of consciousness. On admission, she exhibited abnormal behaviors, including altered speech and shouting. Two days after admission, she lost consciousness and became unresponsive to external stimuli. Her electroencephalogram (EEG) showed generalized rhythmic delta frequency activity at $2-2.5 \mathrm{~Hz}$ with superimposed rhythmic beta frequency activity, which is characteristic of the extreme delta brush pattern. ${ }^{7,8}$ Analysis of her CSF revealed mild pleocytosis $\left(74\right.$ cells $/ \mathrm{mm}^{3}$ ) with a slightly elevated $\mathrm{IgG}$ index (1.01). The timeline of the examination and therapy is shown in Figure 1A.

The patient was diagnosed with anti-NMDAR encephalitis, based on the presence of NMDAR antibodies in her serum and CSF, CSF reactivity in the immunohistochemical analysis using rodent hippocampus (Figure 1B), and bilateral ovarian teratomas on the pelvic computed tomography (CT) findings (Figure 1C).

On the day of admission, she underwent resection of the bilateral teratomas, preserving macroscopically normal ovarian tissue. However, her symptoms were not alleviated

A

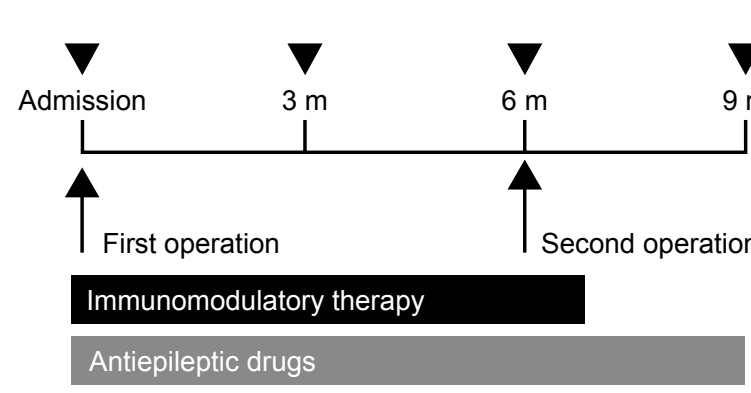

B
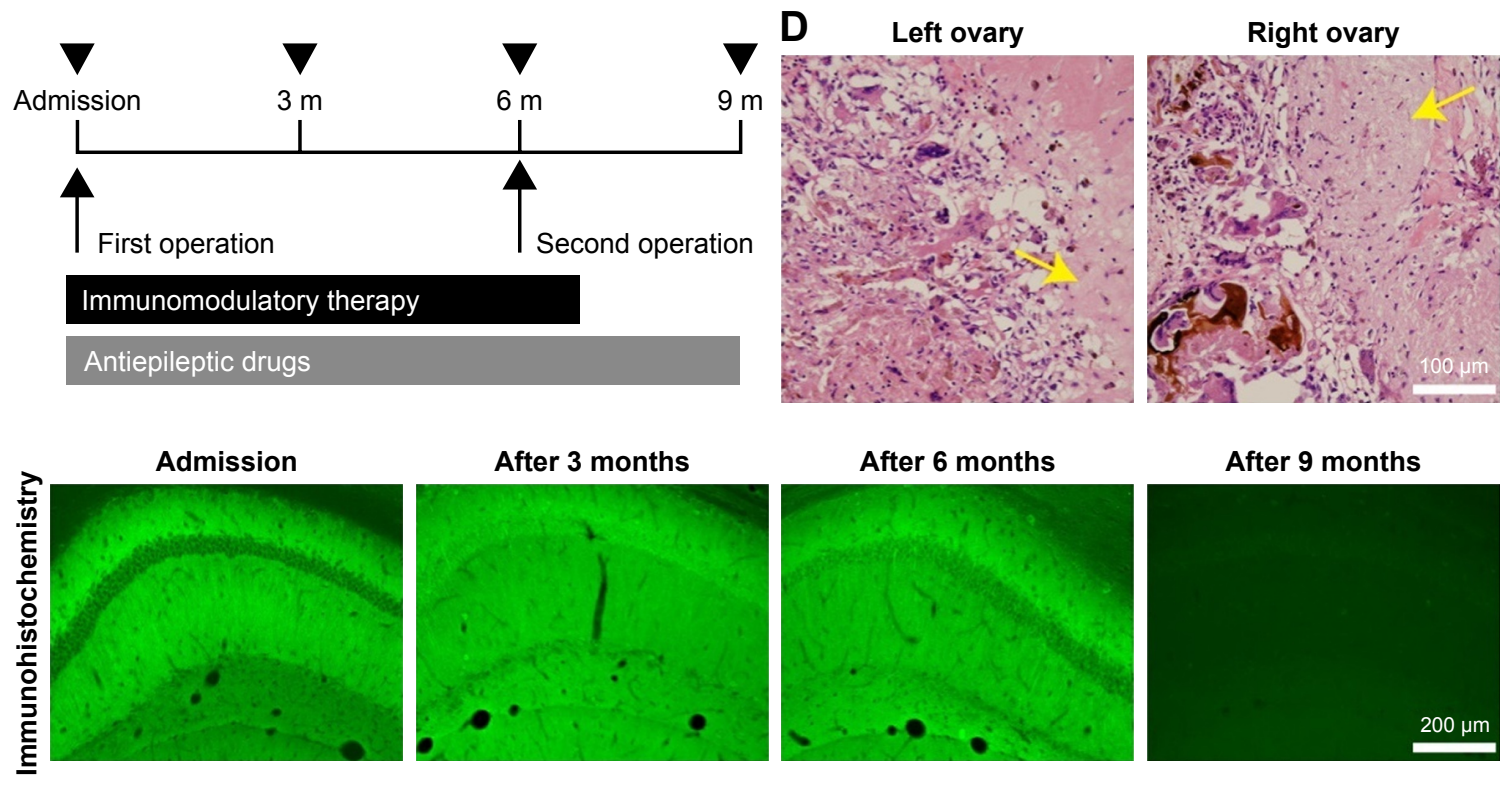

C
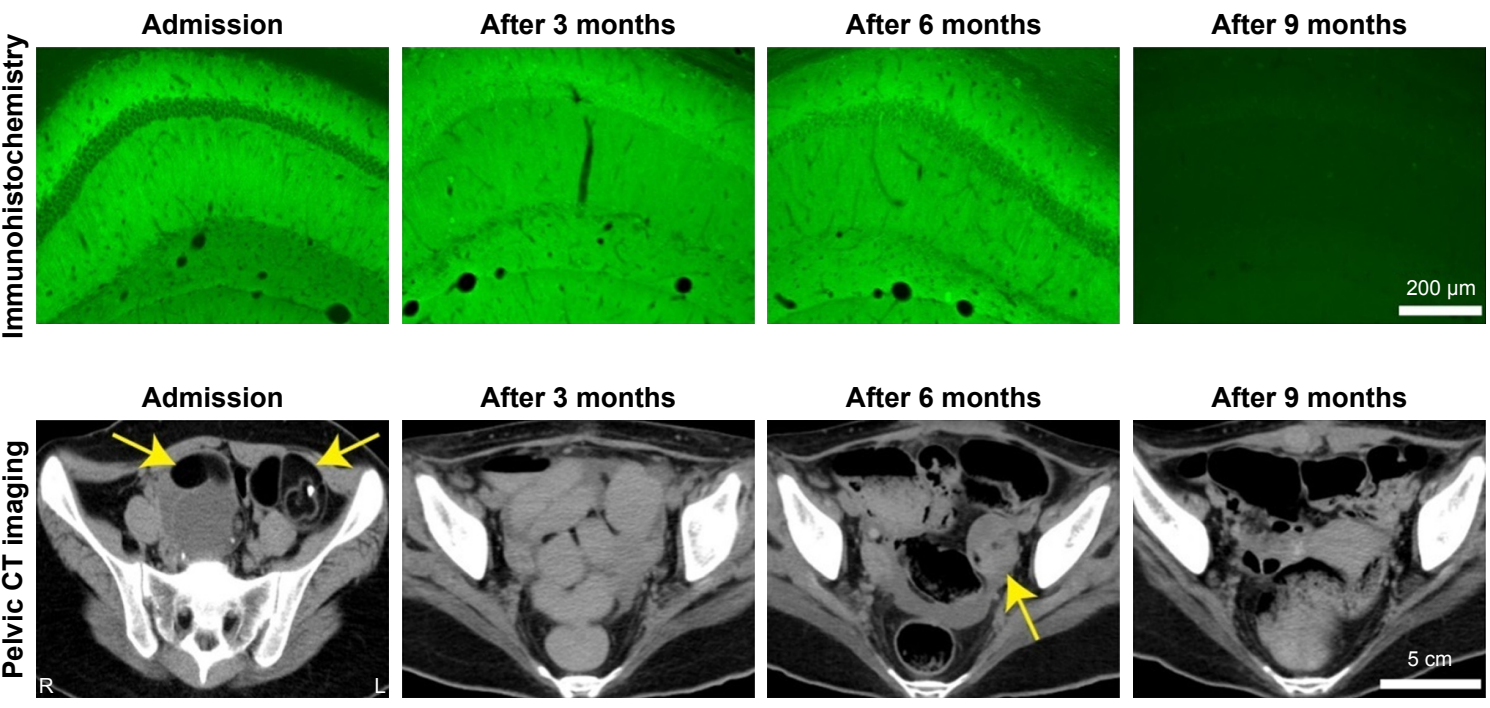

Figure I Clinical course and results of immunohistochemistry, imaging, and pathology analyses.

Notes: (A) Timeline of examination and therapy. Black arrow heads indicate pelvic CT scans and immunohistochemical analyses at 3-month intervals for 9 months. (B) Temporal profile of immunohistochemical analysis of the patient's CSF. Robust reactivity in the hippocampus was observed on admission. This reactivity remained after the first operation and the immunotherapy (after 3 and 6 months). Note that no reactivity was confirmed after the second operation (after 9 months). Scale bar: $200 \mu$ m. (C) Pelvic CT scan images. Bilateral ovarian teratomas on admission (yellow arrows) were enucleated in the first operation. Six months later, recurrence of the left ovarian teratoma could be seen (yellow arrow). The bilateral ovaries were completely resected by salpingo-oophorectomy (second operation; after 9 months). Scale bar: $5 \mathrm{~cm}$. (D) Pathological findings. In addition to the left ovary, the pathological examination of the right ovary showed a mature cystic teratoma, indicating bilateral recurrence of the ovarian teratomas. Yellow arrows show neuroglial tissues. Scale bar: $100 \mu \mathrm{m}$.

Abbreviations: CSF, cerebrospinal fluid; CT, computed tomography; m, months. 
by the surgical excision of the teratomas or subsequent immunotherapies (intravenous methylprednisolone [Solu$\mathrm{Medrol}^{\circledR}$, Pfizer, New York, NY, USA] at 1,000 mg/d over 3 days, intravenous immunoglobulin [Venoglobulin ${ }^{\circledR}$, Japan Blood Products Organization, Tokyo, Japan] at $400 \mathrm{mg} / \mathrm{kg} / \mathrm{d}$ over 5 days, and plasma exchange). Continuous administration of general anesthetic agents, such as midazolam (Midazolam ${ }^{\circledR}$, Sando, Yamagata, Japan), propofol (Propofol ${ }^{\circledR}$, Maruishi, Osaka, Japan), and thiamylal (Isozol ${ }^{\circledR}$, Nichi-Iko Pharmaceutical, Toyama, Japan), was needed to suppress her epileptic activities. Further, several antiepileptic drugs (carbamazepine [Tegretol ${ }^{\circledR}$, Sun Pharma, Mumbai, India], phenobarbital [Phenobal ${ }^{\circledR}$, Fujinaga, Tokyo, Japan], phenytoin [Aleviatin $^{\circledR}$, Dainippon Sumitomo, Osaka, Japan], levetiracetam [E Keppra ${ }^{\circledR}, \mathrm{UCB}$, Brussels, Belgium], and lacosamide $\left[\right.$ Vimpat $\left.^{\circledR}, \mathrm{UCB}\right]$ ) repeatedly induced a generalized rash, which was accompanied by fever, leukopenia, and acute hepatitis, necessitating cessation of these medications.

Although her pelvic CT at 3 months after the first operation showed no signs of tumor, another pelvic CT at 6 months indicated significant recurrence of a left-sided ovarian teratoma (Figure 1C). During her stay in the intensive care unit for 6 months, she remained unconsciousness and her EEG showed generalized rhythmic delta frequency activity. Concurrent immunohistochemical CSF analyses revealed persistently high reactivity of NMDAR antibody (Figure 1B). We thoroughly discussed whether we should remove the bilateral teratomas on the ovaries while saving the normal tissues - ie, similar to the first operation - or completely enucleate the bilateral ovaries, with the medical care team that included neurologists, gynecologists, and intensivists, and the patient's family. Concerned that she might also have a right-sided microscopic ovarian teratoma, the decision was made to perform bilateral salpingo-oophorectomies.

After the second operation, her clinical condition improved rapidly, followed by resolution of her EEG findings. The pathological results showed recurrent mature cystic teratomas on the ovaries from both sides (Figure 1D). The anti-NMDAR reactivity declined within 3 months after the second operation, and the patient was eventually discharged to a rehabilitation facility.

\section{Discussion}

Our patient had an altered mental status that was preceded by a flu-like episode and followed by impaired consciousness. An imaging study revealed bilateral ovarian teratomas, and EEG showed extreme delta brushes, which is characteristic of anti-NMDAR encephalitis. ${ }^{7,8}$ According to the presence of NMDAR antibodies in her serum and CSF, she was diagnosed with anti-NMDAR encephalitis.

Generally, anti-NMDAR encephalitis responds to tumor removal and subsequent immunotherapy, such as intravenous methylprednisolone, intravenous immunoglobulin, and plasma exchange. ${ }^{2,3}$ In this case, however, we wondered why our prompt initiation of intensive treatments had little effect on the disease. Although a second-line therapy, such as rituximab or cyclophosphamide, was considered as bringing about a substantial improvement,, 3 the patient had repeatedly suffered from a generalized rash, which was accompanied by fever, leukopenia, and acute hepatitis, necessitating cessation of additional treatments. During intensive care, we confirmed the persistent high reactivity of NMDAR antibody by immunohistochemical analysis of the CSF. We suspected that we were overlooking a secondary source of immunological activation and repeated the systemic imaging studies, which revealed a recurrent ovarian teratoma 6 months after the first surgical excision of the teratomas. After total enucleation of bilateral ovaries in a second operation, with the significant pathological finding of bilateral mature cystic teratomas, her symptoms improved rapidly, accompanied by a decrease in anti-NMDAR reactivity.

Mature cystic teratomas, frequently called dermoid cysts, are the most common benign germ cell tumors of the ovary in women with anti-NMDAR encephalitis. Although surgical treatment should focus on preserving ovarian tissue, based on fertility being a major concern among young women, the risk of teratoma recurrence should also be discussed. Young patients (age $<30$ years) or those with large $(\geq 8 \mathrm{~cm}$ in diameter) or bilateral cysts are at high risk of recurrence after surgical excision of tumors. ${ }^{10}$ When a patient has all 3 of these factors, as in our case, the recurrence rate is $21.0 \% .{ }^{10}$ In such a patient, physicians should keep in mind the consideration of a recurrent ovarian teratoma if initial treatment fails to induce a clinical improvement. Additionally, gynecologists might not consider recurrence of such benign tumors to be a critical problem, especially when the women are asymptomatic. However, a recurrent ovarian teratoma in patients with antiNMDAR encephalitis must not be overlooked, because it can exacerbate their symptoms. We believe that neurologists have an important role in raising awareness of this situation.

\section{Conclusion}

This case illustrates the need to keep considering why extensive treatment fails to influence the disease when we encounter patients with refractory anti-NMDAR encephalitis, because this diagnosis usually has a favorable prognosis. 
Failure to improve after ovarian resection could be a marker of recurrent ovarian teratoma in anti-NMDAR encephalitis. We emphasize the importance of tumor surveillance repeatedly on the presence of chronic anti-NMDAR reactivity.

\section{Acknowledgment}

Written informed consent has been provided by the patient to have the case details and images published.

\section{Author contributions}

Y Uchida developed the hypotheses, created the figure, and wrote the first draft of the manuscript. D Kato developed the hypotheses and created the figure. Y Yamashita and Y Ozaki analyzed the clinical and pathological data. N Matsukawa developed the hypotheses and reviewed the manuscript. All authors contributed toward data analysis, drafting and critically revising the paper and agree to be accountable for all aspects of the work.

\section{Disclosure}

The authors report no conflicts of interest in this work.

\section{References}

1. Dalmau J, Tüzün E, Wu HY, et al. Paraneoplastic anti-N-methyl-Daspartate receptor encephalitis associated with ovarian teratoma. Ann Neurol. 2007;61:25-36.
2. Dalmau J, Lancaster E, Martinez-Hernandez E, et al. Clinical experience and laboratory investigations in patients with anti-NMDAR encephalitis. Lancet Neurol. 2011;10:63-74.

3. Titulaer MJ, McCracken L, Gabilondo I, et al. Treatment and prognostic factors for long-term outcome in patients with anti-NMDA receptor encephalitis: an observational cohort study. Lancet Neurol. 2013;12:157-165.

4. Iizuka T, Sakai F, Ide T, et al. Anti-NMDA receptor encephalitis in Japan. Long-term outcome without tumor removal. Neurology. 2008; 70:504-511.

5. Gabilondo I, Saiz A, Galán L, et al. Analysis of relapses in anti-NMDAR encephalitis. Neurology. 2011;77:996-999.

6. Thomas A, Rauschkolb P, Gresa-Arribas N, et al. Anti-N-methyl-Daspartate receptor encephalitis: a patient with refractory illness after 25 months of intensive immunotherapy. JAMA Neurol. 2013;70: $1566-1568$.

7. Schmitt SE, Pargeon K, Frechette ES, et al. Extreme delta brush: a unique EEG pattern in adults with anti-NMDA receptor encephalitis. Neurology. 2012;79:1094-1100.

8. Nosadini M, Boniver C, Zuliani L, et al. Longitudinal electroencephalographic (EEG) findings in pediatric anti-N-methyl-D-aspartate (antiNMDA) receptor encephalitis: the Padua experience. J Child Neurol. 2015;30:238-245.

9. Sartori S, Nosadini M, Cesaroni E, et al. Paediatric anti-N-methyl-Daspartate receptor encephalitis: The first Italian multicenter case series. Eur J Paediatr Neurol. 2015;19:453-463.

10. Harada M, Osuga Y, Fujimoto A, et al. Predictive factors for recurrence of ovarian mature cystic teratomas after surgical excision. Eur J Obstet Gynecol Reprod Biol. 2013;171:325-328.
Neuropsychiatric Disease and Treatment

\section{Publish your work in this journal}

Neuropsychiatric Disease and Treatment is an international, peerreviewed journal of clinical therapeutics and pharmacology focusing on concise rapid reporting of clinical or pre-clinical studies on a range of neuropsychiatric and neurological disorders. This journal is indexed on PubMed Central, the 'PsycINFO' database and CAS,

\section{Dovepress}

and is the official journal of The International Neuropsychiatric Association (INA). The manuscript management system is completely online and includes a very quick and fair peer-review system, which is all easy to use. Visit http://www.dovepress.com/testimonials.php to read real quotes from published authors. 